\title{
Design and Implementation of Model Reference Adaptive Controller using Coefficient Diagram Method for a nonlinear process
}

\author{
R.Satheesh Babu ${ }^{1}$, S.Abraham Lincon ${ }^{2}$ \\ ${ }^{1}$ (PG Scholar, Electronics \& Instrumentation Engg, Annamalai University, India) \\ ${ }^{2}$ (Professor, Electronics \& Instrumentation Engg, Annamalai University, India)
}

\begin{abstract}
In this work an adaptive control along with robust control have been developed to address the problem of system performance in the face of system uncertainty in control-system design without excessive reliance on system models. The direct MRAC control scheme with unknowns is designed with control law that is to be combined with an adaptive law. The adaptive laws are developed using the SPR-Lyapunov design approach and are driven by the estimation error for continuous-time systems guaranteeing asymptotic stability of the system states. CDM is a polynomial approach which was developed and introduced for a good transient response of the control systems. An MRAC is constructed in two-degree of-freedom (2DOF) control structure and the adaptation gains of controller parameters are found through CDM. Spherical tank level process is used for validating the design procedure of CDM-MRAC in real time and the performance of controller is compared with linear PI controller. Simulation is carried out using Mat Lab Simulink software.
\end{abstract}

Keywords - Adaptive control, SPR-Lyapunov DMRAC, CDM-MRAC.

\section{INTRODUCTION}

The purpose of feedback control is to achieve desirable system performance in the face of system uncertainty and system disturbances. Although system identification can reduce uncertainty to some extent, residual modelling discrepancies always remain. Controllers must therefore be robust to achieve desired disturbance rejection and/or tracking performance requirements in the presence of such modelling uncertainty. To this end, adaptive control along with robust control have been developed to address the problem of system performance. Adaptive controllers directly or indirectly adjust feedback gains to maintain closed-loop stability and improve performance in the face of system errors. Specifically, indirect adaptive controllers utilize parameter update laws to estimate unknown system parameters and adjust feedback gains to account for system variation, while direct adaptive controllers directly adapt the controller gains in response to system variations. Even though adaptive control algorithms have been developed in the literature for both continuous-time and discrete-time systems, the majority of the discrete-time results are based on recursive least squares and least mean squares algorithms with primary focus on state convergence. Alternatively, Lyapunov-based adaptive controllers have been developed for continuous-time systems guaranteeing asymptotic stability of the system states. In this work, the design and implementation of MRAC using Coefficient diagram method is presented to improve standard designs in adaptive control schemes.

Section 2 describes the design schemes of MRAC for SISO plants, and the development of MRAC for the same plant. Section 3 is devoted to the basics of Coefficient Diagram Method design and development of MRAC using CDM for SISO plants. Section 4 presents the simulation results and comparison of performance of controllers. The conclusion is presented in section 5.

\section{MRAC FOR Siso Plants}

Consider the SISO, LTI plant described by the vector differential equation

$$
\begin{aligned}
& \dot{x}_{p}=A_{p} x_{p}+B_{p} u_{p s} \quad x_{p}(0)=x_{0} \\
& y_{p}=C_{p}^{T} x_{p}
\end{aligned}
$$

Where $x_{p} \in R^{n_{z}} y_{p}, u_{p} \in R^{1}$ and $A_{p}, B_{p}, C_{p}$ have the appropriate dimensions. The transfer function of the plant is given by

$$
y_{p}=G_{p}(s) u_{p}
$$

with $G_{p}(s)$ expressed in the form

$$
G_{p}(s)=k_{p} \frac{Z_{p}(s)}{R_{p}(s)}
$$

where $Z_{p}, R_{p}$ are monic polynomials and $k_{p}$ is a constant referred to as the high frequency gain. The reference model, selected by the designer to describe the desired characteristics of the plant, is described by the differential equation 


$$
\begin{aligned}
& \dot{x}_{m}=A_{m} x_{m}+B_{m} r_{v} \quad x_{m}(0)=x_{m 0} \\
& y_{m}=C_{m}^{T} x_{m}
\end{aligned}
$$

where $x_{m} \in R^{P m}$ for some integer $p_{m}: y_{m}, r \in R^{1}$ and $r$ is the reference input which is assumed to be a uniformly bounded and piecewise continuous function of time. The transfer function of the reference model given by

$$
y_{m}=W_{m}(s) r
$$

Expressed in the same form as (3), i.e.,

$$
W_{m}(s)=k_{m} \frac{Z_{m}(s)}{R_{m}(s)}
$$

Where $Z_{m}(s), R_{m}(s)$ are monic polynomials and $k_{m}$ is a constant.

The MRAC objective is to determine the plant input $\mathrm{u}_{\mathrm{p}}$ so that all signals are bounded and the plant output $y_{p}$ tracks the reference model output $y_{m}$ as close as possible for any given reference input $r(t)$ of the class defined above. If $u_{p}$ is chosen so that the closed-loop transfer function from $r$ to $y_{p}$ has stable poles and is equal to $W_{m}(s)$, the transfer function of the reference model. Such a transfer function matching guarantees that for any reference input signal $r(t)$, the plant output $y_{p}$ converges to $y_{m}$ exponentially fast. This leads to the closed-loop transfer function

$$
\frac{y_{p}}{r}=\frac{k_{m}}{k_{p}} \frac{Z_{m}}{R_{m}} \frac{R_{p}}{Z_{p}} \frac{k_{p} Z_{p}}{R_{p}}=W_{m}(s)
$$

This control law, however, is feasible only when $R_{p}(s)$ is Hurwitz. Otherwise, (6) may involve zero-pole cancellations outside $\mathrm{C}^{-}$, which will lead to unbounded internal states associated with non-zero initial conditions. Consider the feedback control law shown in fig 1.

$$
u_{p}=\theta_{1}^{* T} \frac{\alpha(s)}{\Lambda(s)} u_{p}+\theta_{2}^{* T} \frac{\alpha(s)}{\Lambda(s)} y_{p}+\theta_{a}^{*} y_{p}+c_{0}^{*} r
$$

Where

$$
\begin{array}{ll}
\alpha(s) \triangleq \alpha_{n-2}(s)=\left[s^{n-2}, s^{n-3}{ }_{s, n}, s, 1\right]^{T} & \text { for } n \geq 2 \\
\alpha(s) \triangleq 0 & \text { for } n=1
\end{array}
$$

$c_{0}^{*}, \theta_{a}^{*} \in R^{1} ; \theta_{1}^{*}, \theta_{2}^{*} \in R^{n-1}$ are constant parameters to be designed and $\Lambda(\mathrm{s})$ is an arbitrary monic Hurwitz polynomial of degree $n-1$ that contains $Z_{m}(s)$ as a factor, i.e.,

$$
\Lambda(s)=\Lambda_{0}(s) Z_{m}(s)
$$

Which implies that $\Lambda_{0}(s)$ is monic, Hurwitz and degree $n_{0}=n-1-q_{m}$.

$$
\theta^{*}=\left[\theta_{1}^{*},{ }^{*}, \theta_{2}^{* T}, \theta_{1}^{*}, c_{0}^{*}\right]^{T} \in R^{2 n}
$$

The controller parameter vector is to be chosen so that the transfer function from $r$ to $y_{p}$ is equal to $W_{m}(s)$.

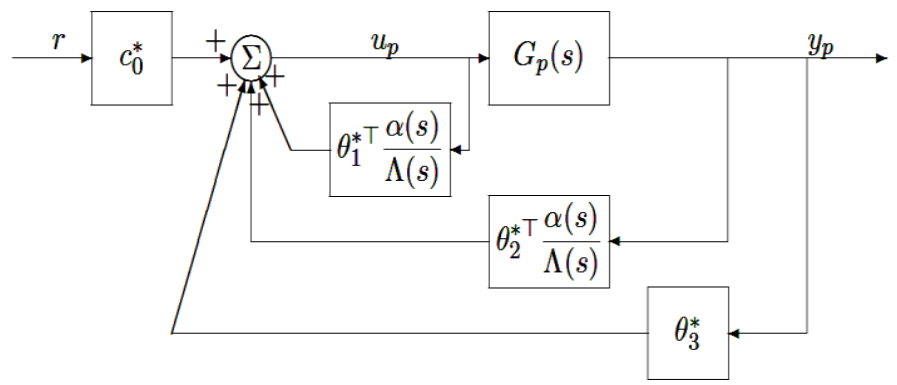

Fig 1 Structure of the MRAC scheme

The I/O properties of the closed-loop plant shown in fig 1 are described by the transfer function equation

$$
y_{\mathrm{p}}=\mathrm{G}_{\mathrm{c}}(\mathrm{s}) r
$$

Where

$$
\mathrm{G}_{\mathrm{c}}(s)=\frac{c_{0}^{*} k_{p} Z_{p} \Lambda^{2}}{\Lambda\left[\left(\Lambda-\theta_{1}^{* T} \alpha(s)\right) R_{p}-k_{p} Z_{p}\left(\theta_{2}^{* T} \alpha(s)+\theta_{a}^{*} \Lambda\right)\right]}
$$

If the controller parameters $\theta_{1}^{*}, \theta_{2}^{*}, \theta_{2}^{*}, c_{0}^{*}$ are selected to meet the control objective so that the closed-loop poles are stable and the closed-loop transfer function $\mathrm{G}_{\mathrm{c}}(\mathrm{s})=W_{m}(s)$, i.e., 


$$
\frac{c_{0}^{*} k_{p} Z_{p} \Lambda^{2}}{\Lambda\left[\left(\Lambda-\theta_{1}^{* T} \alpha(s)\right) R_{p}-k_{p} Z_{p}\left(\theta_{2}^{* T} \alpha(s)+\theta_{a}^{*} \Lambda\right)\right]}=k_{m} \frac{Z_{m}}{R_{m}}
$$

Equation (9) satisfied for all $s \in C$. Because the degree of the denominator of $G_{c}(s)$ is $n_{p}+2 n-2$ and that of $R_{m}(s)$ is $p_{m} \leq n$, for the matching equation to hold, an additional $n_{p}+2 n-2-p_{m}$ zero-pole cancellations must occur in $G_{c}(s)$. Now because $Z_{p}(s)$ is Hurwitz by assumption and $A(s)=\Lambda_{0}(s) Z_{m}(s)$ is designed to be Hurwitz, it follows that all the zeros of $\mathrm{G}_{\mathrm{c}}(\mathrm{s})$ are stable and therefore any zero-pole cancellation can only occur in $\mathrm{C}^{-}$. Choosing

$$
c_{0}^{*}=\frac{k_{m}}{k_{p}}
$$

and using $\Lambda(s)=\Lambda_{0}(s) Z_{m}(s)$ the matching equation (9) becomes

$$
\left(\Lambda-\theta_{1}^{* T} \alpha(s)\right) R_{p}-k_{p} Z_{p}\left(\theta_{2}^{* T} \alpha(s)+\theta_{a}^{*} \Lambda\right)=Z_{p} \Lambda_{0} R_{m}
$$

or

$$
\theta_{1}^{* T} \alpha(s) R_{p}(s)+k_{p}\left(\theta_{2}^{* T} \alpha(s)+\theta_{2}^{*} \Lambda\right) Z_{p}(s)=\Lambda(s) R_{p}(s)-Z_{p}(s) \Lambda_{0}(s) R_{m}(s)
$$

Equating the coefficients of the powers of $s$ on both sides of (12), it can be expressed in terms of the algebraic equation

$$
s \bar{\theta}^{*}=p
$$

Where $\bar{\theta}^{*}=\left[\theta_{1}^{* T}, \theta_{2}^{* T}, \theta_{a}^{*}\right]^{T}, S$ is an $\left(n+n_{p}-1\right) \times(2 n-1)$ matrix that depends on the coefficients of $R_{p}, k_{p} Z_{p}$ and $A$, and $p$ is an $n+n_{p}-1$ vector with the coefficients of $A R_{p}-Z_{p} \Lambda_{0} R_{m}$. The existence of $\bar{\theta}^{*}$ to satisfy (12) and, therefore, (13) will very much depend on the properties of the matrix $S$. For example, if $n>n_{p}$, more than one $\bar{\theta}^{*}$ will satisfy (13), whereas if $n=n_{p}$ and $S$ is nonsingular, equation(12) will have only one solution.

\section{BASICS OF COEFFICIENT DIAGRAM METHOD (CDM)}

To Some mathematical relations extensively used in CDM will be introduced hereafter. The characteristic polynomial is given in the following form

$$
P(s)=a_{n} s^{n}+\cdots+a_{1} s+a_{0} \quad=\sum_{i=0}^{n} a_{i} s^{i}
$$

The stability index $\gamma_{i}$, the equivalent time constant $\tau$, and stability limit $\gamma_{i}^{*}$ are defined as follows.

$$
\begin{array}{ll}
\gamma_{i}=\frac{a_{i}^{2}}{a_{i+1} a_{i-1}}, & i=1 \sim n-1 \\
\tau=\frac{a_{1}}{a_{0}} & \\
\gamma_{i}^{*}=\frac{1}{Y_{i}+1}+\frac{1}{Y_{i}-1}, & \gamma_{n}=\gamma_{0}=\infty
\end{array}
$$

From these equations the following relations are derived.

$$
\begin{aligned}
& \frac{a_{i+1}}{a_{i}}=\frac{\frac{a_{j}}{a_{j-1}}}{Y_{i} Y_{i}-1-Y_{j+1} Y_{j}} \quad i \geq j \\
& a_{\mathrm{i}}=\frac{a_{0} \mathrm{~T}^{i}}{Y_{\mathrm{i}-1} \mathrm{Y}^{2}{ }_{\mathrm{i}-2^{\mathrm{m}} \mathrm{F}_{2}^{i-2} \mathrm{Y}_{1}^{i-1}}}
\end{aligned}
$$

Then characteristic polynomial will be expressed by $a_{0}, \tau$ and $\gamma_{i}$ as follows.

$$
P(s)=a_{0}\left[\left\{\sum_{i=2}^{n}\left(\prod_{j=1}^{i-1} \frac{1}{\gamma_{i-j}^{j}}\right)(\tau s)^{i}\right\}+\tau s+1\right]
$$

The equivalent time constant of the $\mathrm{i}^{\text {th }}$ order $\tau_{\mathrm{i}}$ and the stability index of the $\mathrm{j}^{\text {th }}$ order $\gamma_{\mathrm{i} j}$ are defined as follows.

$$
\begin{aligned}
& \tau_{\mathrm{i}}=\frac{a_{i+1}}{a_{i}}=\frac{\tau}{\gamma_{i} m Y_{2} Y_{1}} \\
& \gamma_{i j}=\frac{a_{i}^{2}}{a_{i+j} a_{i-j}}=\left[\prod_{k=1}^{j-1}\left(\gamma_{i+j-k} \gamma_{i-j-k}\right)^{k}\right] y_{i}^{j}
\end{aligned}
$$

Thus $\tau$ can be considered the equivalent time constant of the 0 -th order and $\gamma_{\mathrm{i}}$ is considered as the stability index of the $1^{\text {st }}$ order. The stability index of the $2^{\text {nd }}$ order is a good measure of stability and is shown below,

$$
\gamma_{i 2}=\frac{\mathbb{a}_{i}^{2}}{a_{i+2} a_{i-2}}=\gamma_{i+1} \gamma_{i}^{2} \gamma_{i-1}
$$

When the performance specifications are given, they must be modified to the design specifications. In $\mathrm{CDM}$, the design specifications are the equivalent time constant $\tau$ and the stability indices $\gamma_{\mathrm{i}}$ for the higher order terms. The stability indices for the lower order terms are already specified. Usually the rise time, the settling time, 
the overshoot, and the peak time are used for the time response specification. However from the CDM design point of view, only the settling time $t_{s}$ is meaningful, because it gives upper bound of $\tau$, where $t_{s}=2.5 \sim 3 \tau$. The frequency response specifications are used for the high frequency attenuation characteristics and the low frequency disturbance rejection characteristics. Using equation (8) the denominator polynomial of $\mathrm{G}_{\mathrm{c}}(\mathrm{s})$ gives the characteristic polynomial of the closed loop system and with the equations (19) and (20) the controller parameters are found.

\section{Simulation Results}

The designed MRAC is implemented in real time also compared with a PI controller. The set point is given in terms of percentage of level. $20 \%$ of level is given as nominal operating value, after 14000(s) the set point has been changed to $30 \%$. The load is applied at the valve in outlet, for 10 liter/min change in outflow. For the sampling time, $1 \mathrm{sec}$ is selected.

Table 1. Plant parameters

\begin{tabular}{|l|c|}
\hline \multicolumn{1}{|c|}{ Process variables } & Nominal operating conditions \\
\hline Level $(\mathrm{h})$ & $1 \mathrm{~m}$ \\
\hline Flow rate, $\left(\mathrm{F}_{\text {in }}\right)$ & $0.2215 \mathrm{~m}^{2} / \mathrm{sec}$ \\
\hline Radius of the tank $(\mathrm{r})$ & $1 \mathrm{~m}$ \\
\hline Constant of the outlet valve $\left(\mathrm{c}_{\mathrm{s}}\right)$ & $0.05 \mathrm{~m}^{2}$ \\
\hline Outlet valve stem position $\left(\mathrm{x}_{\mathrm{s}}\right)$ & 1 \\
\hline Gravitational acceleration $(\mathrm{g})$ & $9.8 \mathrm{~ms}^{-1}$ \\
\hline Maximum level & $2 \mathrm{~m}$ \\
\hline
\end{tabular}

Table 1 provides the description of Spherical tank parameters where table 2 shows the PI controller parameters for various linearized plant models as different operating levels $10 \%, 50 \%$ and $66 \%$ of tank level.

Table 2. PI controller parameters concerning plant models

\begin{tabular}{|c|c|c|c|}
\hline Linearized models & Transfer function models & \multicolumn{2}{|c|}{ PI Controller parameters } \\
\cline { 3 - 4 } & & $\mathrm{K}_{\mathrm{c}}$ & $\mathrm{K}_{\mathrm{i}}$ \\
\hline Model 1 & $\frac{4 e^{-225 s}}{440 s+1}$ & 0.825 & 0.002 \\
\hline Model 2 & $\frac{6 e^{-2 x s s}}{1200 s+1}$ & 1.385 & 0.003 \\
\hline Model 3 & $\frac{275 e^{-125 s}}{1050 s+1}$ & 2.29 & 0.005 \\
\hline
\end{tabular}

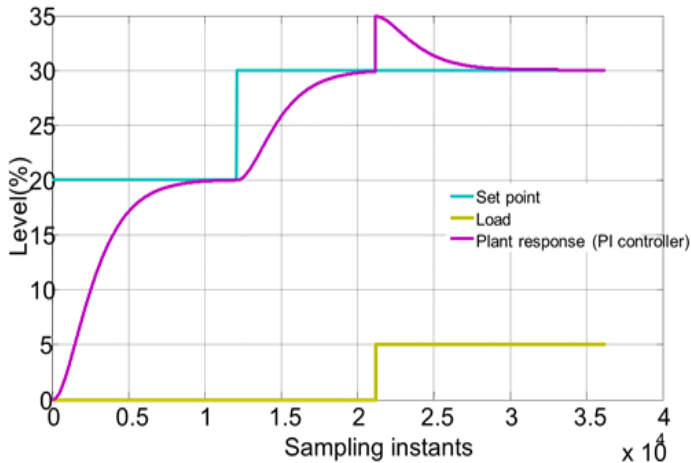

Fig 2. Plant response with PI controller

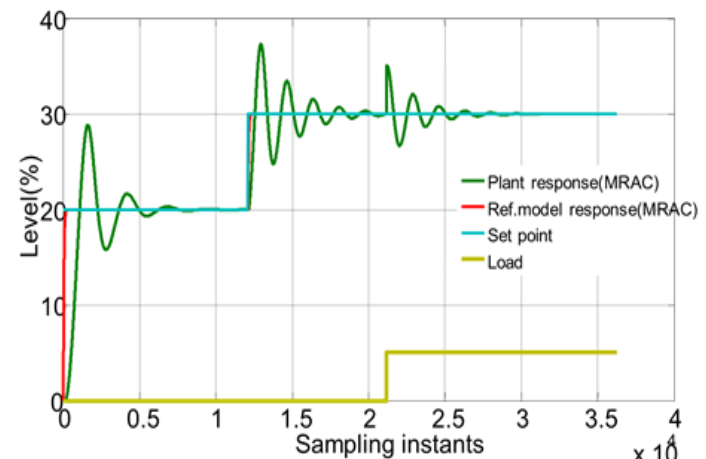

Fig 3. Plant response with CDM-MRAC

Fig 2 shows the closed loop response of plant with PI controller. Notice that the response has large rise time and settling time. Fig 3 shows that the response of plant with MRAC. Notice that the rise time has increased as the reference model has high rise time also the settling time. 


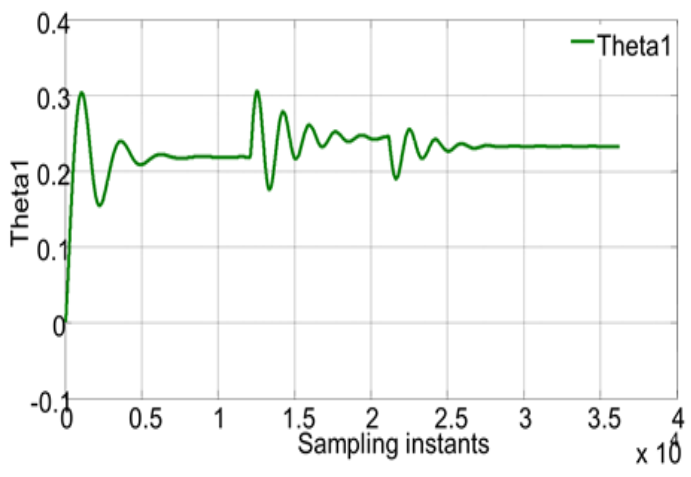

Fig 4. MRAC controller parameter theta1

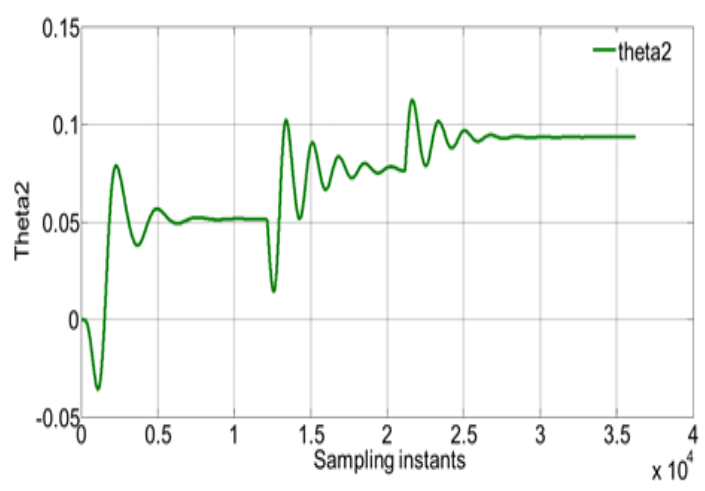

Fig 5. MRAC controller parameter theta2

Fig 4 and 5 show that the convergence of controller parameters as the tracking error goes asymptotically zero. And fig 6 shows the comparison of performance between the PI controller and CDM-MRAC.

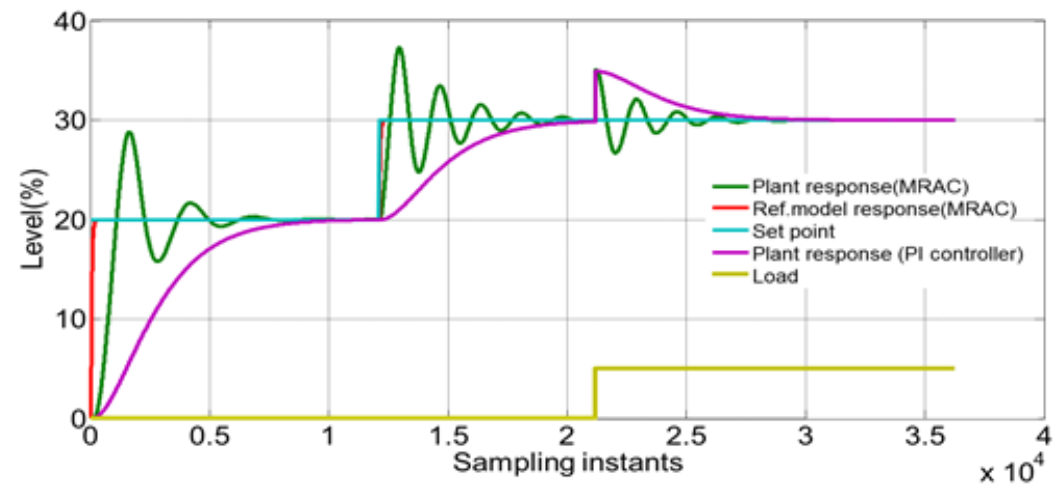

Fig 6. Comparison of plant responses with CDM-MRAC and PI controller

Table 3. Comparison of controllers performance in time domain

\begin{tabular}{|c|c|c|c|c|}
\hline \multicolumn{2}{|c|}{ Controller structure } & Settling time (ts) secs & \% MP & Rise time (t $\mathbf{t}_{\mathbf{r}}$ ) secs \\
\hline \multirow{4}{*}{ MRAC using CDM } & Servo 1 & 12500 & 48 & 2000 \\
\cline { 2 - 5 } & Servo 2 & 1200 & 50 & 500 \\
\cline { 2 - 5 } & Load 1 & 1100 & - & - \\
\hline \multirow{3}{*}{ PI controller } & Servo 1 & 12500 & - & 1200 \\
\cline { 2 - 5 } & Servo 2 & 1200 & - & 1100 \\
\cline { 2 - 5 } & Load 1 & 1100 & - & - \\
\hline
\end{tabular}

Table 4. Comparison of controllers performance

\begin{tabular}{|l|l|l|l|}
\hline Controller structure & ISE & IAE & ITAE \\
\hline CDM-MRAC & $5.504 \mathrm{e}+5$ & $7.699 \mathrm{e}+4$ & $1.078 \mathrm{e}+9$ \\
\hline PI controller & $1.65 \mathrm{e}+6$ & $1.717 \mathrm{e}+5$ & $2.442 \mathrm{e}+9$ \\
\hline
\end{tabular}

From fig 6, Table 3 and 4 provides the various performance specifications in time domain between CDMMRAC and PI controller. From these, the CDM-MRAC outperforms the PI controller. 
V. Hardware Implementation

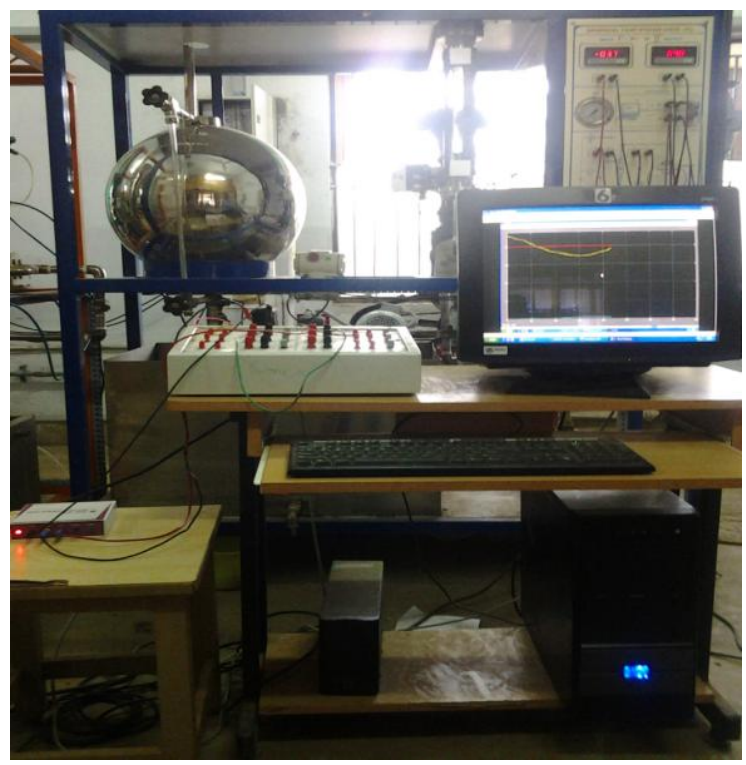

Fig 7. Closed loop apparatus setup of Spherical tank level process (Real time)

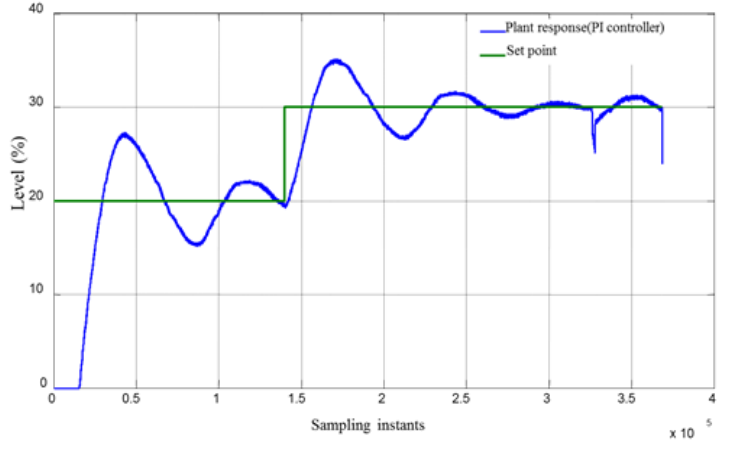

Fig 8. Spherical tank response of PI controller in real time

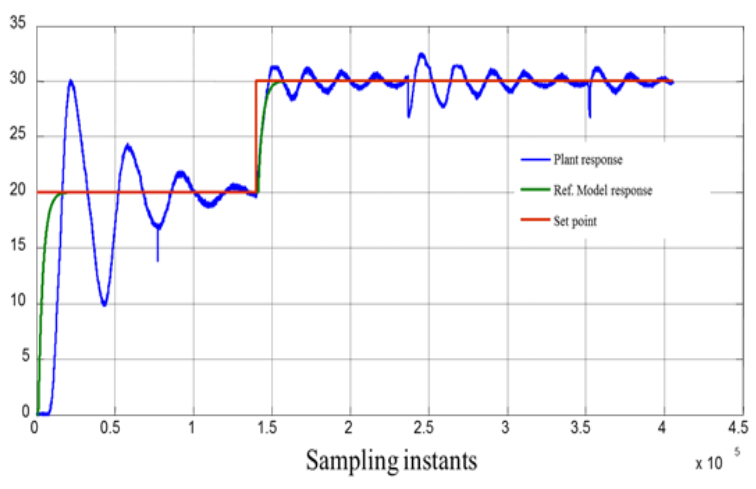

Fig 9.Spherical tank response of CDMMRAC in real time

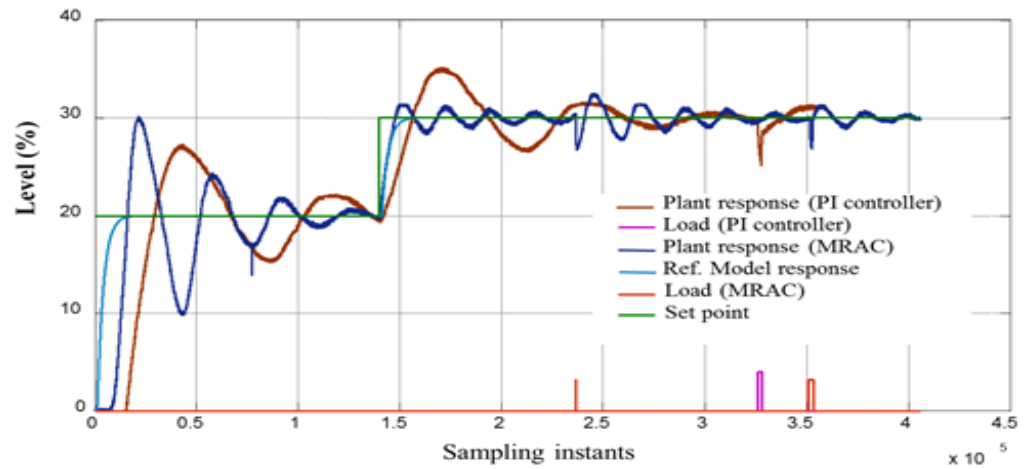

Fig 10. Comparison of Plant responses of CDM-MRAC and PI controller

Fig 8 shows the closed loop response of plant with PI controller and fig 9 gives the closed loop response of plant with CDM-MRAC scheme. Notice that from Fig 10, it shows that the MRAC provides improved rise time and settling time and it has $50 \%$ of overshoot. The response with PI controller provides less rise time and settling 
time and $30 \%$ of overshoot. And the servo and regulatory responses are good in MRAC scheme. The following tables 5 and 6 show that the comparison of various performance specifications in time domain.

Table 5. Performance comparison of controllers in real time

\begin{tabular}{|c|c|c|c|c|}
\hline \multicolumn{2}{|c|}{ Controller structure } & $\begin{array}{c}\text { Settling time } \\
(\mathbf{m s})\end{array}$ & $\%$ MP & $\begin{array}{c}\text { Rise time } \\
\left(\mathbf{t}_{\mathbf{r}}\right)\end{array}$ \\
\hline \multirow{4}{*}{$\begin{array}{c}\text { MRAC using } \\
\text { CDM }\end{array}$} & Servo 1 & $1.2 \mathrm{e}+5$ & 40 & $1.6 \mathrm{e}+4$ \\
\cline { 2 - 5 } & Servo 2 & $8 \mathrm{e}+5$ & 10 & $1.5 \mathrm{e}+4$ \\
\cline { 2 - 5 } & Load 1 & $1.4 \mathrm{e}+5$ & - & - \\
\cline { 2 - 5 } & Load 2 & $4.8 \mathrm{e}+4$ & - & - \\
\hline \multirow{4}{*}{ PI controller } & Servo 1 & NA & 32 & $2.9 \mathrm{e}+4$ \\
\cline { 2 - 5 } & Servo 2 & $1.6 \mathrm{e}+5$ & 50 & $1.6 \mathrm{e}+4$ \\
\cline { 2 - 5 } & Load 1 & NA & - & - \\
\hline
\end{tabular}

Table 6. Comparison of controllers performance

\begin{tabular}{|c|c|c|}
\hline Controller structure & ISE & IAE \\
\hline CDM-MRAC & 4532492 & 24.83563 \\
\hline PI controller & 10532359 & 25.74069 \\
\hline
\end{tabular}

\section{Conclusion}

The model reference control is designed for the stability of a closed loop system in the sense of Lyapunov. From the closed loop transfer function, the characteristics polynomial has been taken, the CDM is applied on the characteristics polynomial to find the unknown adaptation gains. The strength of CDM lies in that, for any plant, minimum phase or non-minimum phase, the simplest and robust controller under practical limitation can be found. Such controller closely agrees with the controllers which are accepted as good controllers in practical application.

\section{REFERENCES}

[1] S. Manabe. Coefficient Diagram Method. Automatic Control in Aerospace, Seoul, Korea. 211-222, 1998.

[2] S. Manabe. A simple proof and the physical interpretation of Routh stability criterion. J. IEEE Japan, 117, (12), 851-854, 1997c.

[3] Petros A. Ioannou and Jing Sun. Robust Adaptive control, 8-12, 105-132, 330-363.

[4] Karl Johan Astrom and Bjorn Wittenmark. Adaptive control, second edition. 210-15.

[5] Pavan K. Vempaty, Ka C. Cheok, and Robert N. K. Loh. Experimental Implementation of Lyapunov based MRAC for Small Biped Robot Mimicking Human Gait. ISA Transactions- 21(3), 2007.

[6] Muhammad Nasiruddin Mahyuddin and Mohd Rizal Arshad. Performance Evaluation of Direct Model Reference Adaptive Control on a Coupled-tank Liquid Level System. Elektrika-10(2), 2008.

[7] Serdar Ethem Hamamci Nusret Tan. Design of PI controllers for achieving time and frequency domain specifications simultaneously. ISA Transactions- 45(4), 2006. 